\title{
EXTENDIBILITY, MONODROMY, AND LOCAL TRIVIALITY FOR TOPOLOGICAL GROUPOIDS
}

\author{
OSMAN MUCUK and ILHAN IÇEN
}

(Received 11 September 2000 and in revised form 26 February 2001)

\begin{abstract}
A groupoid is a small category in which each morphism has an inverse. A topological groupoid is a groupoid in which both sets of objects and morphisms have topologies such that all maps of groupoid structure are continuous. The notion of monodromy groupoid of a topological groupoid generalizes those of fundamental groupoid and universal cover. It was earlier proved that the monodromy groupoid of a locally sectionable topological groupoid has the structure of a topological groupoid satisfying some properties. In this paper a similar problem is studied for compatible locally trivial topological groupoids.
\end{abstract}

2000 Mathematics Subject Classification. 22A05, 22A22, 58H05.

1. Introduction. Let $G$ be a topological groupoid and $W$ an open subset of $G$ containing all the identities of $G$. Then the monodromy groupoid $M(G, W)$ of $G$ is defined as in Definition 2.5, which is due to Pradines [13]. In Mucuk [12] (see also Brown and Mucuk $[5,6]$ for Lie groupoid case) in the case where $G$ is locally sectionable, it was proved that the groupoid $M(G, W)$ may be given the structure of a topological groupoid such that each star $M(G, W)_{x}$ is a universal cover of $G_{x}$ and $M(G, W)$ has a universal property on the globalization of continuous pregroupoid morphisms to topological groupoid morphisms.

If $X$ is a topological space, then $G=X \times X$ is a topological groupoid with the groupoid multiplication $(x, y)(y, z)=(x, z)$. If $X$ is a path connected topological space which has a universal cover, then the monodromy groupoid of the topological groupoid $G=X \times X$, for a suitable open subset $W$ of $G$, is the fundamental groupoid $\pi_{1}(X)$.

If $G$ is a topological group which can be thought of as a topological groupoid with only one object such that it has a universal cover, then for a suitable open subset $W$ of $G$ the monodromy groupoid $M(G, W)$ is just the universal cover of $G$.

Thus the concept of the monodromy groupoid generalizes the notions of the fundamental groupoid as a topological groupoid and the universal cover.

In Mackenzie [11] the monodromy groupoid denoted by $\Pi G$ of a topological groupoid $G$, in which each star $G_{x}$ has a universal cover, is constructed directly from the universal covers of $G_{x}$ 's, and then $\Pi G$ is given a topology under some conditions studied in terms of principal groupoid.

In this paper, we define compatible locally trivial groupoid and similar to the fundamental groupoid case studied by Brown and Danish in [3] prove that the monodromy groupoid of a compatible locally trivial topological groupoid can be topologised with an appropriate topology. 
2. Groupoids and topological groupoids. A groupoid $G$ on $O_{G}$ is a small category in which each morphism is an isomorphism. The explicit definition is as follows.

DEFINITION 2.1. A groupoid $G$ has a set of morphisms which we call just elements of $G$, a set $O_{G}$ of objects together with maps $\alpha, \beta: G \rightarrow O_{G}$ and $\epsilon: O_{G} \rightarrow G$ such that $\alpha \epsilon=\beta \epsilon=1_{O_{G}}$, the identity map. The maps $\alpha, \beta$ are called initial and final point maps, respectively, and the map $\epsilon$ is called object inclusion. If $a, b \in G$ and $\beta(a)=\alpha(b)$, then the multiplication $a b$ exists such that $\alpha(a b)=\alpha(a)$ and $\beta(a b)=\beta(b)$. So there exists a partial multiplication defined by the map $G_{\beta} \times{ }_{\alpha} G \rightarrow G,(a, b) \mapsto a b$, where

$$
G_{\beta} \times{ }_{\alpha} G=\{(a, b) \in G \times G: \beta(a)=\alpha(b)\} .
$$

Further, this partial multiplication is associative, for $x \in O_{G}$ the element $\epsilon(x)$ denoted by $1_{x}$ acts as the identity, and each element $a$ has an inverse $a^{-1}$ such that $\alpha\left(a^{-1}\right)=$ $\beta(a), \beta\left(a^{-1}\right)=\alpha(a), a a^{-1}=(\epsilon \alpha)(a), a^{-1} a=(\epsilon \beta)(a)$. The map $G \rightarrow G, a \mapsto a^{-1}$ is called the inversion.

Let $G$ be a groupoid. We write $O_{G}$ for the set of objects of $G$, and also identify $O_{G}$ with the set of identities of $G$. An element of $O_{G}$ may be written as $x$ or $1_{x}$ as convenient. We write $G(x, y)$ for $\alpha^{-1}(x) \cap \beta^{-1}(x)$ if $x, y \in O_{G}$, where $\alpha^{-1}(x)$ (resp., $\beta^{-1}(x)$ ) is called star (resp., costar) of $x$ and denoted by $G_{x}$ (resp., by $G^{x}$ ).

A subgroupoid of $G$ is a pair of subsets $H \subseteq G$ and $O_{H} \subseteq O_{G}$ such that $\alpha(H) \subseteq O_{H}$, $\beta(H) \subseteq O_{H}, 1_{x} \in H$ for each $x \in O_{H}$ and $H$ is closed under the partial multiplication and inversion in $G$.

Let $G$ and $H$ be groupoids. A morphism from $H$ to $G$ is a functor, that is, it consists of a pair of functions $f: H \rightarrow G$ and $O_{f}: O_{H} \rightarrow O_{G}$ preserving all the structures.

DEFINITION 2.2 (see [11]). A topological groupoid is a groupoid $G$ on $O_{G}$ together with topologies on $G$ and $O_{G}$ such that the maps which define the groupoid structure are continuous, namely the initial and final point maps $\alpha, \beta: G \rightarrow O_{G}$, the object inclusion map $\epsilon: O_{G} \rightarrow G, x \mapsto \epsilon(x)$, the inversion $G \rightarrow G, a \mapsto a^{-1}$, and the map $G_{\beta} \times_{\alpha} G \rightarrow G,(a, b) \mapsto a b$, where $G_{\beta} \times_{\alpha} G$ has the subspace topology from $G \times G$.

A morphism of topological groupoids $f: H \rightarrow G$ is a morphism of groupoids in which both maps $f: H \rightarrow G$ and $O_{f}: O_{H} \rightarrow O_{G}$ are continuous.

Note that in this definition the map $G_{\beta} \times_{\alpha} G \rightarrow G,(a, b) \mapsto a b$ and the inversion map $G \rightarrow G, a \mapsto a^{-1}$ are continuous if and only if the map $\delta: G \times_{\alpha} G \rightarrow G,(a, b) \mapsto a^{-1} b$, called the groupoid difference map, is continuous, where

$$
G \times{ }_{\alpha} G=\{(a, b) \in G \times G: \alpha(a)=\alpha(b)\}
$$

has the subspace topology from $G \times G$. Again if one of the maps $\alpha, \beta$, and the inversion are continuous, then the other one is continuous.

Let $X$ be a topological space. Then $G=X \times X$ is a topological groupoid on $X$, in which each pair $(x, y)$ is considered as a morphism from $x$ to $y$ and the partial multiplication is defined by $(x, y) \circ(y, z)=(x, z)$. The inverse of $(x, y)$ is $(y, x)$ and the identity $1_{x}$ at $x \in O_{G}$ is the pair $(x, x)$.

Note that in a topological groupoid $G$, for each $a \in G(x, y)$ right translation $R_{a}$ : $G^{x} \rightarrow G^{y}, b \mapsto b a$ and left translation $L_{a}: G_{y} \rightarrow G_{x}, b \mapsto a b$ are homeomorphisms. 
DEFINITION 2.3. A groupoid $G$, in which each star $G_{x}$ (and hence costar $G^{x}$ ) has a topology such that for $a \in G(x, y)$ the right translation $R_{a}: G^{x} \rightarrow G^{y}, b \mapsto b a$ (and hence the left translation $\left.L_{a}: G_{y} \rightarrow G_{x}, b \mapsto a b\right)$ is a homeomorphism, is called a star topological groupoid.

DeFinITION 2.4. Let $G$ be a star topological groupoid. A subset $V$ of $G$ is called star connected (resp., star path connected) if for each $x \in O_{G}$, the star $V_{x}=V \cap G_{x}$ of $V$ at $x$ is connected (resp., path connected). So a star topological groupoid $G$ is star connected (resp., star path connected) if each star $G_{x}$ of $G$ is connected (resp., path connected).

Let $G$ be a groupoid and let $W$ be any subset of $G$ such that $X=O_{G} \subseteq W$. Then $W$ obtains the structure of pregroupoid: this means that $W$ is a graph, in the sense that it has the structure of maps $\alpha_{W}, \beta_{W}: W \rightarrow X, \imath: X \rightarrow W$ with $\alpha_{W} \imath=\beta_{W} \imath=1_{X}$, and further there is a partial multiplication on $W$ in which if $a b$ is defined then $\beta_{W}(a)=\alpha_{W}(b)$, $\left(\imath \beta_{W} a\right) a=a,\left(\imath \alpha_{W} a\right) a=a$, and each $a \in W$ has an inverse $a^{-1}$ such that $a a^{-1}=\imath \alpha_{W} a$, $a^{-1} a=\imath \beta_{W} a$. There is also an associativity condition. For further discussion of this, see, for example, Crowell and Smythe [7]. For our purpose we do not need this, since we know already that $W$ is embeddable in a groupoid.

We follow the method of Brown and Mucuk [5], which generalizes the work for groups in Douady and Lazard [8].

There is a standard construction $M(G, W)$ associating to the pregroupoid $W$ and there is a pregroupoid morphism $\tilde{\imath}: W \rightarrow M(G, W)$ which is universal for pregroupoid morphisms to a groupoid. First we form the free groupoid $F(W)$ on the graph $W$, and denote the inclusion $W \rightarrow F(W)$ by $a \mapsto[a]$. Let $N$ be the normal subgroupoid (see Higgins [10] and Brown [2]) of $F(W)$ generated by the elements $[a][b][a b]^{-1}$ for all $a, b \in W$ such that $a b$ is defined and belongs to $W$. Then $M(G, W)$ is defined to be the quotient groupoid $F(W) / N$. The composition $W \rightarrow F(W) \rightarrow M(G, W)$ is written $\tilde{\imath}$, and is the required universal morphism. It follows that $\tilde{\imath}$ is injective.

The map $\tilde{i}$ has a universal property that if $f: W \rightarrow H$ is a pregroupoid morphism then there is a morphism of groupoids $f^{\prime}: M(G, W) \rightarrow H$ such that $f=f^{\prime} \tilde{i}$. In particular the inclusion map $i: W \rightarrow G$ globalises to a morphism of groupoids $p: M(G, W) \rightarrow G$ called canonical morphism.

We give this construction as a definition.

DEFINITION 2.5. Let $G$ be a topological groupoid and $W$ an open subset of $G$ such that $O_{G} \subseteq W$. Let $F(W)$ be the free groupoid on $W$ and $N$ the normal subgroupoid of $F(W)$ generated by the elements in the form $[a][b][a b]^{-1}$ for $a, b \in W$ such that $a b$ is defined and $a b \in W$. Then the quotient groupoid $F(W) / N$ is called monodromy groupoid of $G$ for $W$ and denoted by $M(G, W)$.

3. Monodromy groupoids of locally sectionable topological groupoids. In this section we recall some results from Brown and Mucuk [5].

Let $G$ be a topological groupoid such that each star $G_{x}$ has a universal cover. The groupoid $\Pi G$ as the union of the universal covers of $G_{x}$ 's is defined as follows. As a set, $\Pi G$ is the union of the stars $\left(\pi_{1} G_{x}\right)_{1_{x}}$. The object set of $\Pi G$ is the same as that 
of $G$. The function $\alpha: \Pi G \rightarrow X$ maps all of $\left(\pi_{1} G_{x}\right)_{1_{x}}$ to $x$, while $\beta: \Pi G \rightarrow X$ is on $\left(\pi_{1} G_{X}\right)_{1}$, the composition of the two final point maps

$$
\left(\pi_{1} G_{X}\right)_{1_{X}} \rightarrow G \rightarrow X
$$

As explained in Mackenzie [11, page 67], there is a multiplication on $\Pi G$ given by "concatenation," that is,

$$
[a] \circ[b]=[a+a(1) b],
$$

where the $(+)$ inside the bracket denotes the usual composition of paths. Here $a$ is assumed to be a path in $G_{x}$ from $1_{x}$ to $a(1)$, where $\beta(a(1))=y$, say, so that $b$ is a path in $G_{y}$, and for each $t \in[0,1]$, the product $a(1) b(t)$ is defined in $G$, yielding a path $b(a(1))$ from $a(1)$ to $a(1) b(1)$. It is straightforward to prove that in this way $\Pi G$ becomes a groupoid, and that the final map of paths induces a morphism of groupoids $p: \Pi G \rightarrow G$.

DEFINITION 3.1. Let $X$ be a topological space admitting a simply connected cover. A subset $U$ of $X$ is called liftable if $U$ is open, path connected and the inclusion $U \rightarrow X$ maps each fundamental group of $U$ trivially. If $U$ is liftable, and $q: Y \rightarrow X$ is a covering map, then for any $y \in Y$ and $x \in U$ such that $q y=x$, there is a unique map $\hat{\imath}: U \rightarrow Y$ such that $\hat{\imath} x=y$ and $q \hat{\imath}$ is the inclusion $U \rightarrow X$.

THEOREM 3.2. Suppose that $G$ is a star connected star topological groupoid and $W$ is an open neighborhood of $O_{G}$ satisfying the condition:

$W$ is star path connected and $W^{2}$ is contained in a star path connected neighborhood $V$ of $O_{G}$ such that for all $x \in O_{G}, V_{x}$ is liftable, where

$$
W^{2}=W W=\{a b: \beta(a)=\alpha(b)\} .
$$

Then there is an isomorphism of star topological groupoids $M(G, W) \rightarrow \Pi G$, and hence the morphism $p: M(G, W) \rightarrow G$ is a star universal covering map.

The following definition is due to Ehresmann [9].

DeFinItion 3.3. Let $G$ be a groupoid and $X=O_{G}$ be a topological space. An admissible local section of $G$ is a function $s: U \rightarrow G$ from an open subset $U$ of $X$ such that

(1) $\alpha s(x)=x$ for all $x \in U$,

(2) $\beta s(U)$ is open in $X$, and

(3) $\beta s$ maps $U$ homeomorphically to $\beta s(U)$.

Let $W$ be a subset of $G$ such that $X \subseteq W$ and let $W$ have the structure of a topological space. We say that $(\alpha, \beta, W)$ is locally sectionable if for each $w \in W$ there is an admissible local section $s: U \rightarrow G$ of $G$ such that

(i) $s \alpha(w)=w$

(ii) $s(U) \subseteq W$, and

(iii) $s$ is continuous as a function from $U$ to $W$.

Such an $s$ is called a continuous admissible local section. 
Let $G$ be a groupoid and $W$ a subset of $G$ containing all the identities of $G$. Then we say $W$ generates $G$ as a groupoid if each element of $G$ can be written as a multiplication of some elements of $G$.

The following definition is due to Pradines [13] under the name "morceau de groupoide différentiables."

DEFINITION 3.4. A locally topological groupoid is a pair $(G, W)$ consisting of a groupoid $G$ and a topological space $W$ such that

$\left(G_{1}\right) \quad O_{G} \subseteq W \subseteq G$,

$\left(G_{2}\right) \quad W=W^{-1}$,

$\left(G_{3}\right)$ the set $W(\delta)=\left(W \times_{\alpha} W\right) \cap \delta^{-1}(W)$ is open in $W \times_{\alpha} W$ and the restriction of $\delta$ to $W(\delta)$ is continuous,

$\left(G_{4}\right)$ the restrictions to $W$ of the initial and final point maps $\alpha$ and $\beta$ are continuous and the triple $(\alpha, \beta, W)$ is locally sectionable,

$\left(G_{5}\right) \quad W$ generates $G$ as a groupoid.

Note that, in this definition, $G$ is a groupoid but does not need to have a topology. The locally topological groupoid $(G, W)$ is said to be extendible if there can be found a topology on $G$ making it a topological groupoid and for which $W$ is an open subset. In general $(G, W)$ is not extendible, but there is a holonomy groupoid $\operatorname{Hol}(G, W)$ and a morphism $\phi: \operatorname{Hol}(G, W) \rightarrow G$ of groupoids such that $\operatorname{Hol}(G, W)$ admits the structure of topological groupoid. The construction is given in details in Aof and Brown [1]. For an example of locally topological groupoid which is not extendible see [1].

THEOREM 3.5 (globalisability theorem). Let $(G, W)$ be a locally topological groupoid. Then there is a topological groupoid $H$, a morphism $\phi: H \rightarrow G$ of groupoids, and an embedding $i: W \rightarrow H$ of $W$ to an open neighborhood of $O_{H}$ such that the following conditions are satisfied:

(i) $\phi$ is the identity on objects, $\phi i=i d_{W}, \phi^{-1}(W)$ is open in $H$, and the restriction $\phi_{W}: \phi^{-1}(W) \rightarrow W$ of $\phi$ is continuous,

(ii) if $A$ is a topological groupoid and $\xi: A \rightarrow G$ is a morphism of groupoids such that

(a) $\xi$ is the identity on objects,

(b) the restriction $\xi_{W}: \xi^{-1}(W) \rightarrow W$ of $\xi$ is smooth and $\xi^{-1}(W)$ is open in $A$ and generates $A$,

(c) the triple $\left(\alpha_{A}, \beta_{A}, A\right)$ is locally sectionable,

then there is a unique morphism $\xi^{\prime}: A \rightarrow H$ of topological groupoids such that $\phi \xi^{\prime}=\xi$ and $\xi^{\prime} a=i \xi a$ for $a \in \xi^{-1}(W)$.

The groupoid $H$ is called the holonomy groupoid $\operatorname{Hol}(G, W)$ of the locally topological groupoid $(G, W)$.

Let $G$ be a topological groupoid on $X$. Then $G$ is called locally trivial if for each $x \in X$ there is an open set $U$ containing $x$ and a section $s: U \rightarrow G_{X}$ of $\beta$. Thus $\beta s=1_{U}$ and for each $y \in U, \alpha(s(y))=x$, that is, $s(y): x \rightarrow y$ in $G$.

The following result, whose proof is due to Pradines, is given in [1]. In the proof of this result the sectionable condition is used. 
Proposition 3.6 (see [1, Proposition 6.1]). A locally trivial locally topological groupoid is extendible.

As a corollary of Theorem 3.5, the following result is obtained. See Brown and İçen [4] for a nice application of this result to the holonomy groupoids of local subgroupoids.

COROLlARY 3.7. Let $G$ be a topological groupoid and $p: M \rightarrow G$ a morphism of groupoids such that $p: O_{M} \rightarrow O_{G}$ is the identity. Let $W$ be an open subset of $G$ such that

(a) $O_{G} \subseteq W$,

(b) $W=W^{-1}$,

(c) $W$ generates $G$,

(d) $\left(\alpha_{W}, \beta_{W}, W\right)$ is locally sectionable,

and suppose that $\tilde{\imath}: W \rightarrow M$ is given such that $p \tilde{\imath}=i: W \rightarrow G$ is the inclusion and $W^{\prime}=\tilde{i}(W)$ generates $M$.

Then $M$ admits a unique structure of topological groupoid such that $W^{\prime}$ is an open subset and $p: M \rightarrow G$ is a morphism of topological groupoids mapping $W^{\prime}$ homeomorphically to $W$.

These imply Theorem 3.10 which is called the strong monodromy principle, namely the globalization of continuous local morphisms to continuous morphisms on the monodromy groupoid.

THEOREM 3.8. Let $G$ be a locally sectionable topological groupoid and $W$ an open subset of $G$ containing $O_{G}$, such that $W=W^{-1}$, and $W$ generates $G$. Then the monodromy groupoid $M=M(G, W)$ admits the structure of topological groupoid such that $\tilde{l}(W)$ is an open subspace of $M$ and any continuous pregroupoid morphism on $W$ globalises to a continuous morphism on $M$.

THEOREM 3.9. Suppose further to the assumptions of Theorem 3.8 that $G$ is star path connected, that each star $G_{x}$ has a simply connected cover, and that $W^{2}$ is contained in an open neighborhood $V$ of $O_{G}$ such that each star $V_{x}$ in $G_{x}$ is liftable. Then the projection $p: M(G, W) \rightarrow G$ is the universal covering map on each star, and so $M(G, W)$ is isomorphic to the star universal cover $\Pi G$ of $G$.

THEOREM 3.10 (strong monodromy principle). Let $G$ be a locally sectionable star path connected topological groupoid and $W$ a neighborhood of $O_{G}$ in $G$ such that $W$ satisfies the condition:

$W$ is path connected and $W^{2}$ is contained in a star path connected neighborhood $V$ of $O_{G}$ such that for all $x \in O_{G}, V_{x}$ is liftable.

Let $f: W \rightarrow H$ be a continuous pregroupoid morphism from $W$ to the topological groupoid $H$. Then $f$ determines uniquely a morphism $f^{\prime}: \Pi G \rightarrow H$ of topological groupoids such that $f^{\prime} j^{\prime}=f$.

4. Extendibility of compatible locally trivial groupoids. The concept of locally triviality is due to Ehresmann [9]. It is also used by Mackenzie in [11]. We adapt this concept as follows. 
Definition 4.1. Let $X$ be a topological space and $G$ a groupoid on $X$. Let $u=$ $\left\{U_{i}: i \in I\right\}$ be an open cover of $X$ which is also a base for $X$ such that if $x \in U_{i}$ then there is a local section $s_{x, i}: U_{i} \rightarrow G_{x}$ of $\beta$ such that $s_{x, i}(x)=x$. Thus for $y \in U_{x, i}$, $s_{x, i}(y): x \rightarrow y$ in $G$. Such a map $s_{x, i}: U_{i} \rightarrow G_{x}$ is called local section about $x \in O_{G}$. $G$ is called compatible locally trivial if the following compatible condition is satisfied.

Cомp 4.2. For $x \in X$, if $s_{x, i}: U_{i} \rightarrow G_{x}$ and $s_{x, j}: U_{j} \rightarrow G_{x}$ are two local sections about $x$, then there is an open neighborhood $V_{i j}$ of $x$ in $U$ such that $V_{i j} \subseteq U_{i} \cap U_{j}$ and $s_{x, i}\left|V_{i j}=s_{x, j}\right| V_{i j}$.

If $s_{x, i}: U_{i} \rightarrow G_{x}$ is a local section about $x$, then we write $\tilde{U}_{x, i}$ for the image $s_{x, i}\left(U_{i}\right)$. We now prove the following theorem on the extendibility of compatible locally trivial groupoid to a topological groupoid. This result is similar to the fundamental groupoid case studied earlier in [3].

THEOREM 4.3. Let $G$ be a compatible locally trivial groupoid. Let $a \in G(x, y)$. Then the sets $\left(\tilde{U}_{x, i}\right)^{-1} a\left(\tilde{U}_{y, j}\right)$ for all $x \in U_{i}$ and $y \in U_{j}$ form a set of basic neighborhoods for a topology on $G$ such that $G$ is a topological groupoid with this topology.

Proof. By the compatibility condition it is obvious that these sets form a topology on $G$. Because if $\left(\tilde{U}_{x, i}\right)^{-1} a\left(\tilde{U}_{y, j}\right)$ and $\left(\tilde{U}_{x, i^{\prime}}\right)^{-1} a\left(\tilde{U}_{y, j^{\prime}}\right)$ are basic open neighborhood of $a$, then $x \in U_{i} \cap U_{i^{\prime}}$ and $y \in U_{j} \cap U_{j^{\prime}}$. By the compatible condition there is a basic open neighborhood $V_{i i^{\prime}}$ of $x$ in $U$ such that $V_{i i^{\prime}} \subseteq U_{i} \cap U_{i^{\prime}}$ and $s_{x, i}\left|V_{i i^{\prime}}=s_{x, i^{\prime}}\right| V_{i i^{\prime}}$. Similarly there is a basic open neighborhood $V_{j j^{\prime}}$ of $y$ such that $V_{j j^{\prime}} \subseteq U_{j} \cap U_{j^{\prime}}$ and $s_{x, j}\left|V_{i j}=s_{x, j^{\prime}}\right| V_{j j^{\prime}}$. Hence $\left(\tilde{V}_{x, i i^{\prime}}\right)^{-1} a\left(\tilde{V}_{y, j j^{\prime}}\right)$ is a basic open neighborhood of $a$ such that

$$
\left(\tilde{V}_{x, i i^{\prime}}\right)^{-1} a\left(\tilde{V}_{y, j j^{\prime}}\right) \subseteq\left(\tilde{U}_{x, i}\right)^{-1} a\left(\tilde{U}_{y, j}\right) \cap\left(\tilde{U}_{x, i^{\prime}}\right)^{-1} a\left(\tilde{U}_{y, j^{\prime}}\right) .
$$

We now prove that $G$ is a topological groupoid with this topology. To prove that the groupoid difference map

$$
\delta: G \times{ }_{\alpha} G \longrightarrow G, \quad(a, b) \longmapsto a^{-1} b
$$

is continuous, let $\delta(a, b)=a^{-1} b$, where $a \in G(x, y)$ and $b \in G(x, z)$. Let $\left(\tilde{U}_{y, j}\right)^{-1} a^{-1}$ $b\left(\tilde{U}_{z, k}\right)$ be a basic neighborhood of $a^{-1} b$. Then $\left(\tilde{U}_{x, i}\right)^{-1} a\left(\tilde{U}_{y, j}\right) \times_{\alpha}\left(\tilde{U}_{x, i}\right)^{-1} b\left(\tilde{U}_{z, k}\right)$ is an open neighborhood of $(a, b)$ and

$$
\delta\left(\left(\tilde{U}_{x, i}\right)^{-1} a\left(\tilde{U}_{y, j}\right) \times_{\alpha}\left(\tilde{U}_{x, i}\right)^{-1} b\left(\tilde{U}_{z, k}\right)\right)=\left(\tilde{U}_{y, j}\right)^{-1} a^{-1} b\left(\tilde{U}_{z, k}\right) .
$$

So $\delta$ is continuous.

To prove the continuity of the final point map $\beta: G \rightarrow X$, let $a \in G$ with $\alpha(a)=x$ and $\beta(a)=y$, and let $U_{j}$ be a basic open neighborhood of $y$ in $u$. Then $\left(\tilde{U}_{x, i}\right)^{-1} a\left(\tilde{U}_{y, j}\right)$ is a basic open neighborhood of $a$ and $\beta\left(\left(\tilde{U}_{x, i}\right)^{-1} a\left(\tilde{U}_{y, j}\right)\right) \subseteq U_{j}$. Hence $\beta$ is continuous. Further since $\delta$ and $\beta$ are both continuous so also is the initial point map $\alpha: G \rightarrow X$.

Finally for the continuity of the object inclusion $\epsilon: X \rightarrow G$, let $x \in X$ and let $\left(\tilde{U}_{x, i}\right)^{-1} 1_{x}\left(\tilde{U}_{x, j}\right)$ be a basic open neighborhood of $1_{x}$. Then $x \in U_{i} \cap U_{j}$, and by the compatibility condition there is an open neighborhood of $x$ in $u$ such that $V_{i j} \subseteq U_{i} \cap U_{j}$ and $s_{x, i}\left|V_{i j}=s_{x, j}\right| V_{i j}$. So $\epsilon\left(V_{i, j}\right) \subseteq\left(\tilde{U}_{x, i}\right)^{-1} 1_{x}\left(\tilde{U}_{x, j}\right)$. Hence $\epsilon$ is continuous. 
In particular in this result if we take $G$ to be the fundamental groupoid $\pi_{1} X$ on a topological space $X$ which is locally nice, then we obtain a result given in [3] stated as follows.

EXAMPLE 4.4. Let $X$ be a locally path connected and semilocally 1-connected topological space. Then there is an open cover $U$ of $X$ as in Definition 4.1 consisting of all open, path connected subsets $U$ of $X$ such that there is only one homotopy class of the paths in $U$ between same points. If $x \in U_{i}$ for $U_{i} \in U$, then the local section $s_{x, i}: U_{i} \rightarrow \pi_{1} X$ is defined by choosing for each $y \in U_{i}$ a path in $U_{i}$ from $x$ to $y$ and taking $s_{x, i}(y)$ to be the homotopy class of this path. Since the path class of the paths in $U_{i}$ between same points is unique, the local section $s_{x, i}$ is well defined. Further since $X$ is locally path connected, the compatibility condition is satisfied. Thus $\pi_{1} X$ is a compatible locally trivial groupoid.

Let $G$ be a topological groupoid and $W$ an open neighborhood of $O_{G}$ such that $O_{G} \subseteq$ $W$. Then we have the monodromy groupoid $M(G, W)$ defined as in Definition 2.5. Note that by the construction of $M(G, W)$ we have a pregroupoid morphism $\tilde{\imath}: W \rightarrow M(G, W)$ which is an inclusion map. Let $\tilde{W}$ be the image of $W$ under this inclusion and let $\tilde{W}$ have the topology such that $\tilde{\imath}: W \rightarrow \tilde{W}$ is a homeomorphism. Then we have the following result.

Proposition 4.5. Let $G$ be a topological groupoid whose underlying groupoid is compatible locally trivial and $W$ an open subset of $G$ such that $O_{G} \subseteq W$. Suppose that each local section $s_{x, i}: U_{x, i} \rightarrow G_{x}$ has image in $W$, that is, $s_{x, i}\left(U_{x, i}\right) \subseteq W$. Then the groupoid $M(G, W)$ is compatible locally trivial.

Proof. The proof is immediate since $W$ is isomorphic to $\tilde{W}$ and the inclusion $\tilde{\imath}: W \rightarrow \tilde{W}$ is the identity on objects.

As a corollary of this result we state the following.

COROLLARY 4.6. Let $G$ be a topological groupoid whose groupoid is compatible locally trivial and $W$ an open subset of $G$ including all the identities. Suppose that each local section $s_{x, i}: U_{x, i} \rightarrow G_{x}$ has image in $W$. Then the monodromy groupoid $M(G, W)$ has the structure of a topological groupoid.

We now give a result which is similar to Theorem 4.3 under strong conditions.

THEOREM 4.7. Let $X$ be a topological space and $G$ a groupoid on $X$. Let $W$ be a compatible locally trivial subgroupoid of $G$. Then $G$ has the structure of a topological groupoid such that $W$ is open in $G$.

Proof. By Theorem 4.3, $G$ has the structure of a topological groupoid. Let $a \in W$ with $\alpha(a)=x$ and $\beta(b)=y$. Since for $x \in O_{G}, s_{x, i}(x)=1_{x},\left(\tilde{U}_{x, i}\right)^{-1} a\left(\tilde{U}_{y, j}\right)$ is an open neighborhood of $a$ and since $W$ is a subgroupoid, $\left(\tilde{U}_{x, i}\right)^{-1} a\left(\tilde{U}_{y, j}\right) \subseteq W$. So $W$ is open in $G$.

COROLLARY 4.8. Let $G$ be a topological groupoid and $W$ a compatible locally trivial subgroupoid of $G$. Then the monodromy groupoid $M(G, W)$ admits the structure of a topological groupoid such that $\tilde{\imath}(W)=\tilde{W}$ is open in $M(G, W)$, and any continuous 
pregroupoid morphism $f: W \rightarrow H$ globalises to a morphism of topological groupoids $\tilde{f}: M(G, W) \rightarrow H$.

Proof. Since $\tilde{\imath}: W \rightarrow \tilde{W}$ is a homeomorphism and $O_{G}=O_{M(G, W)}, \tilde{W}$ is a compatible locally trivial subgroupoid of $M(G, W)$. So by Theorem $4.7, M(G, W)$ becomes a topological groupoid such that $\tilde{W}$ is open in $M(G, W)$. Let $f: W \rightarrow H$ be a continuous pregroupoid morphism. By the universal property of $M(G, W), f$ globalises to a morphism $\tilde{f}: M(G, W) \rightarrow H$ which is continuous on $\tilde{W}$. But $\tilde{W}$ is open in $M(G, W)$ and $\tilde{W}$ generates $M(G, W)$. So the continuity of $\tilde{f}$ on $M(G, W)$ follows.

THEOREM 4.9. Let $G$ be a topological groupoid which is compatible locally trivial such that each star $G_{x}$ has a universal cover and let $W$ be an open subset of $G$ such that $O_{G} \subseteq W$ and $W^{2}$ is contained in an open neighborhood $V$ of $O_{G}$ such that each star $V_{x}$ is liftable. Suppose that each local section $s_{x, i}: U_{x, i} \rightarrow G_{x}$ has image in $W$. Then the monodromy groupoid $M(G, W)$ may be given a topological groupoid structure such that each star $M(G, W)_{x}$ is isomorphic to the universal cover of $G_{x}$.

Proof. By Proposition 4.5, the groupoid $M(G, W)$ is compatible locally trivial, and by Theorem 4.3 it becomes a topological groupoid. Further, by Theorem 3.2, we identify $M(G, W)$ with $\Pi G$, the union of all the universal covers of the stars $G_{x}$ 's.

As a corollary of these results we give the following.

THEOREM 4.10 (monodromy principle). Let $G$ be a topological groupoid in which each star $G_{x}$ is path connected and has a universal cover and $W$ a locally compatible subgroupoid of $O_{G}$ such that $W$ is star liftable. Then any continuous pregroupoid morphism $f: W \rightarrow H$ determines uniquely a morphism $f^{\prime}: \Pi G \rightarrow H$ of topological groupoids such that $f^{\prime} \tilde{j}=f$.

ACKNOWLEDGEMent. We would like to thank Prof. Ronald Brown for introducing this area to us, and to the referees for their helpful comments.

\section{REFERENCES}

[1] M. A. E.-S. A.-F. Aof and R. Brown, The holonomy groupoid of a locally topological groupoid, Topology Appl. 47 (1992), no. 2, 97-113. MR 94e:22008. Zbl 855.22007.

[2] R. Brown, Topology: A Geometric Account of General Topology, Homotopy Types and the Fundamental Groupoid, Ellis Horwood Series in Mathematics and its Applications, Ellis Horwood, Chester, 1988. Zbl 655.55001.

[3] R. Brown and G. Danesh-Naruie, The fundamental groupoid as a topological groupoid, Proc. Edinburgh Math. Soc. (2) 19 (1975), 237-244. MR 54\#1217. Zbl 297.55001.

[4] R. Brown and İ. İçen, Lie local subgroupoids and their holonomy and monodromy Lie groupoids, Topology Appl. 115 (2001), no. 2, 125-138. CMP 1847459.

[5] R. Brown and O. Mucuk, The monodromy groupoid of a Lie groupoid, Cahiers Topologie Géom. Différentielle Catég. 36 (1995), no. 4, 345-369. MR 97b:22002. Zbl 844.22006.

[6]___ Foliations, locally Lie groupoids and holonomy, Cahiers Topologie Géom. Différentielle Catég. 37 (1996), no. 1, 61-71. MR 97j:22005. Zbl 868.22001.

[7] R. H. Crowell and N. Smythe, Congruences and geometric amalgamations of groupoids, Houston J. Math. 13 (1987), no. 4, 499-525. MR 89e:20099. Zbl 654.20064.

[8] A. Douady and M. Lazard, Espaces fibrés en algèbres de Lie et en groupes, Invent. Math. 1 (1966), 133-151. MR 33\#5787. Zbl 144.01804. 
[9] C. Ehresmann, Catégories topologiques et catégories différentiables, Colloque Géom. Diff. Globale (Bruxelles, 1958), Centre Belge Rech. Math., Louvain, 1959, pp. 137-150 (French). MR 22\#7148. Zbl 205.28202.

[10] P. J. Higgins, Notes on Categories and Groupoids, Van Nostrand Rienhold Mathematical Studies, vol. 32, Van Nostrand Reinhold, London, 1971. MR 48\#6288. Zbl 226.20054 .

[11] K. Mackenzie, Lie groupoids and Lie Algebroids in Differential Geometry, London Mathematical Society Lecture Note Series, vol. 124, Cambridge University Press, Cambridge, 1987. MR 89g:58225. Zbl 683.53029.

[12] O. Mucuk, Covering groups of non-connected topological groups and the monodromy groupoid of a topological groupoid, Ph.D. thesis, University of Wales, 1993.

[13] J. Pradines, Théorie de Lie pour les groupoïdes différentiables. Relations entre propriétés locales et globales, C. R. Acad. Sci. Paris Sér. A-B 263 (1966), A907-A910 (French). MR 35\#4954. Zbl 147.41102.

OSMAN MucuK: DePARTMENT OF MATHEMATICS, FACUlTy OF SCIENCE AND ART, ERCIYES UNIVERSITY, KAYSERI, TURKEY

E-mail address: mucuk@erciyes.edu.tr

ILHAN IÇEN: DePARTMENT OF MATHEMATICS, FACUlTy OF SCIENCE AND ART, INÖNÜ UNIVERSITY, MALATYA, TURKEY

E-mail address: i icen@inonu.edu.tr 


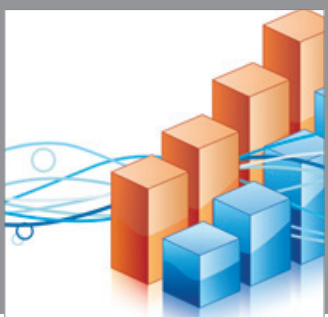

Advances in

Operations Research

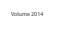

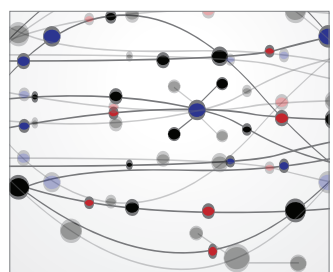

\section{The Scientific} World Journal
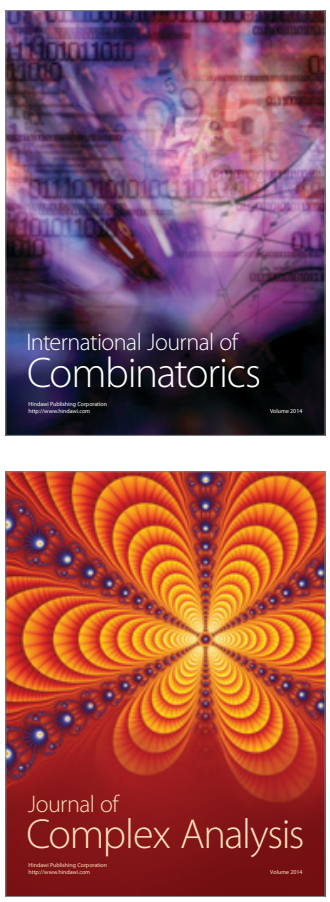

International Journal of

Mathematics and

Mathematical

Sciences
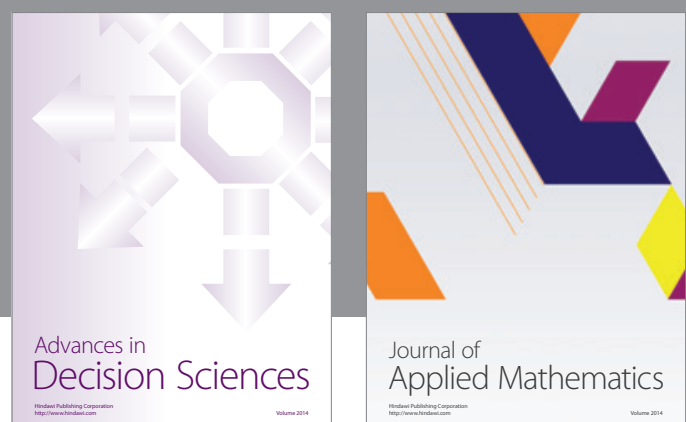

Journal of

Applied Mathematics
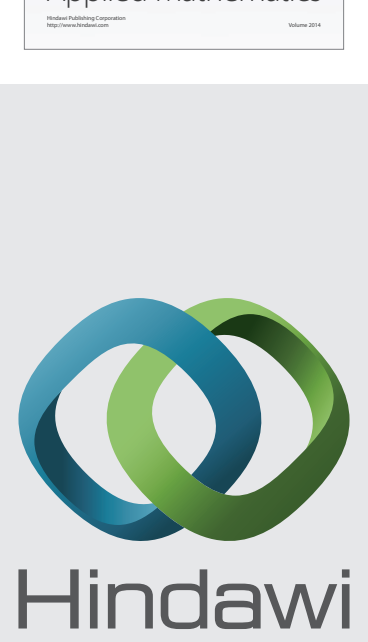

Submit your manuscripts at http://www.hindawi.com
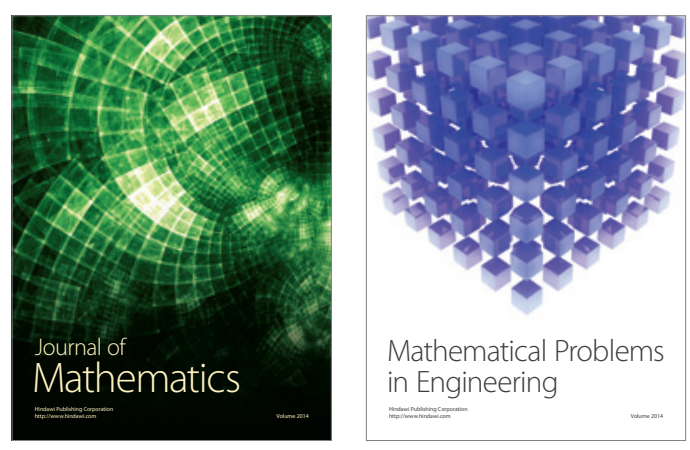

Mathematical Problems in Engineering
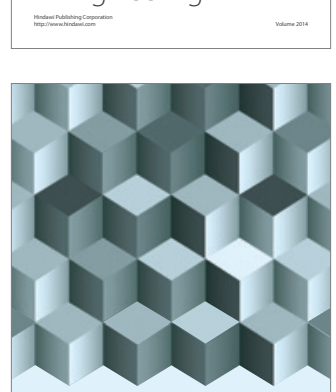

Journal of

Function Spaces
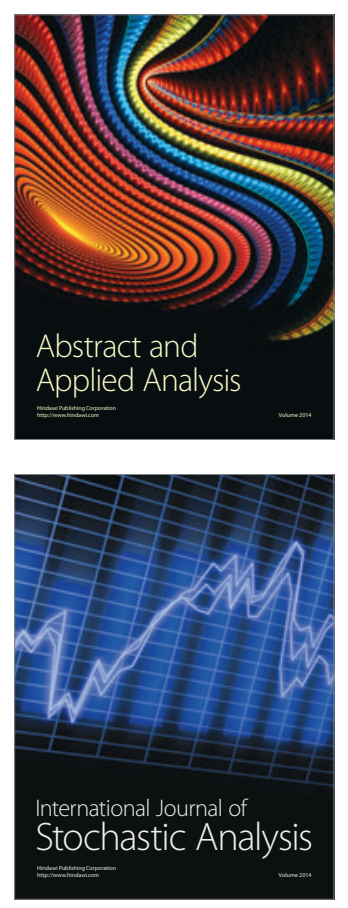

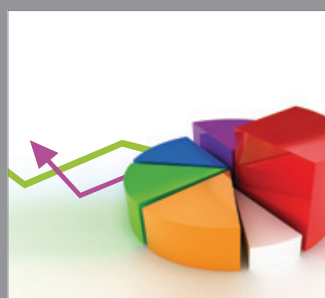

ournal of

Probability and Statistics

Promensencen
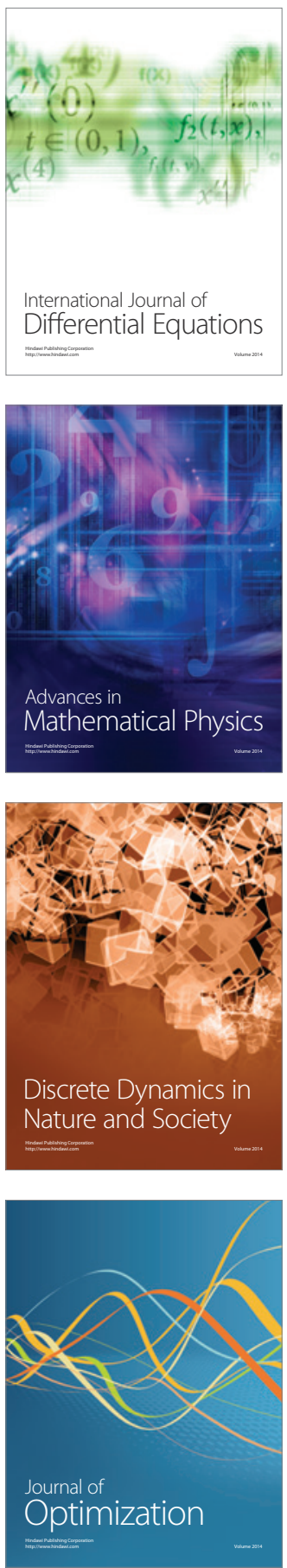